\title{
Transfixion incision as an initial technique in nasal tip deprojection
}

\author{
Philip Solomon MD FRCSC ${ }^{1,2,3,4}$, Richard Rival MD FRCSC 1,2 , Aimee Mabini MD FRCSC ${ }^{2}$, Jennifer Boyd RN BScN 5
}

P Solomon, R Rival, A Mabini, J Boyd. Transfixion incision as an initial technique in nasal tip deprojection. Can J Plast Surg 2008;16(4):224-227.

BACKGROUND: The overprojected nasal tip presents a significant challenge to the surgeon performing rhinoplasty. Full transfixion incision as a means of dealing with this deformity has been studied only in combination with other surgical methods.

OBJECTIVES: To determine whether transfixion incision alone would result in significant nasal tip deprojection, and if skin thickness had an effect on the extent of deprojection the procedure yielded.

METHOD: Seventy-two consecutive patients with an aesthetic goal of nasal tip deprojection were enrolled. The sole surgical means of deprojection used was transfixion incision. Subjects were categorized as thin-, medium- or thick-skinned based on the surgeon's analysis. These groups were compared in terms of their postprocedural nasal tip deprojection.

RESULTS: Using transfixion incision as the sole means of correcting this deformity resulted in a mean nasal tip deprojection of $1.6 \mathrm{~mm}$ (ranging from $0 \mathrm{~mm}$ to $3 \mathrm{~mm}$ ). Patients in the thin skin group had a mean deprojection of $2.12 \mathrm{~mm}$. This was significantly greater than for both the mediumand thick-skinned groups. There was no significant difference between the medium- and thick-skinned groups.

CONCLUSIONS: Surgeons may use skin thickness when planning interventions for correcting nasal tip overprojection. When used alone, transfixion incision resulted in tip deprojection comparable with that achieved when combined with other methods, particularly for thin-skinned patients. Surgeons can thus use a graduated approach in which transfixion incision, the least destructive method, is used before proceeding with other interventions.

Key Words: Nasal tip deprojection; Over-projected nasal tip; Pinocchio deformity; Rhinoplasty; Transfixion incision

$\mathrm{T}$ he overprojected nasal tip, commonly referred to as the 'Pinocchio' nose, is a relatively uncommon but challenging deformity (1). Surgical correction of the nasal tip is the most difficult and the least predictable component of rhinoplasty surgery. The goal of the nasal aesthetic surgeon is to use his or her judgment to try and achieve the patient's goals and optimize the aesthetics of the nose.

Nasal tip projection is important for aesthetic and functional reasons. It is cosmetically important for facial harmony but also functionally important for adequate air entry. The patient's aesthetic goal must be carefully balanced with the maintenance of functional support because any procedure that reduces tip projection narrows the nasal valve and reduces air flow. The perception of nasal tip projection is influenced by many factors other than nasal anatomy. These include chin

\section{Une incision transfixiante comme technique initiale pour diminuer la projection de la pointe du nez}

HISTORIQUE : Une pointe du nez trop projetée représente un grand défi pour le chirurgien qui procède à une rhinoplastie. On n'a jamais étudié la possibilité d'une incision transfixiante complète pour traiter cette malformation autrement qu'en association avec d'autres méthodes chirurgicales.

OBJECTIFS : Déterminer si l'incision transfixiante seule entraînerait une diminution notable de la projection de la pointe du nez et si l'épaisseur de la peau a un effet sur l'importance de cette diminution.

MÉTHODOLOGIE : Soixante-douze patients consécutifs ayant un objectif esthétique de diminution de la projection de la pointe du nez ont participé à l'étude. L'incision transfixiante a constitué la seule méthode chirurgicale à cet effet. Les sujets ont été classés comme ayant la peau mince, la peau moyenne ou la peau épaisse selon l'analyse du chirurgien. On a comparé ces groupes d'après la diminution de la projection de la pointe du nez après l'intervention.

RÉSULTATS : L'utilisation de l'incision transfixiante comme seul moyen de corriger cette malformation a favorisé une diminution de la projection moyenne de la pointe du nez de 1,6 $\mathrm{mm}$ (plage de $0 \mathrm{~mm}$ à $3 \mathrm{~mm}$ ). Les patients ayant la peau mince présentaient une diminution moyenne de 2,12 $\mathrm{mm}$. Ce résultat était considérablement plus important que dans les groupes ayant la peau moyenne ou épaisse. On ne constatait pas de différence significative entre ces deux groupes.

CONCLUSIONS : Les chirurgiens peuvent tenir compte de l'épaisseur de la peau lorsqu'ils planifient des interventions pour corriger des pointes du nez trop projetées. Utilisée seule, l'incision transfixiante favorisait une diminution de la projection de la pointe du nez comparable à celle obtenue au moyen d'autres méthodes combinées, notamment chez les patients ayant la peau mince. Les chirurgiens peuvent donc utiliser une démarche graduée, dans le cadre de laquelle ils font appel à l'incision transfixiante, la moins destructive, avant d'entreprendre d'autres interventions.

projection, upper lip height, nasolabial angle, dorsal height, nasofrontal angle and other physical characteristics (2).

Nasal tip support has typically been classified as either major or minor. Major tip-support mechanisms were first described by Janeke and Wright (3) and have been further studied. The 'tripod concept' was then described, which relates the medial and lateral crura to projection and rotation of the nasal tip (4,5). Generally, major tip support includes the size, shape and resiliency of medial and lateral crura; the attachment of the medial crural footplates to the caudal septum; and soft-tissue attachment of the caudal margin of the upper lateral cartilages to the cephalic margin of the lower lateral cartilages (2). In addition, several minor tip-support mechanisms have been described, including the dorsal cartilaginous septum, the interdomal ligaments, the membranous septum, the nasal

\footnotetext{
${ }^{1}$ Department of Otolaryngology-Head and Neck Surgery, University of Toronto; ${ }^{2}$ St Michael's Hospital, Toronto; ${ }^{3}$ York Central Hospital, Richmond Hill;

${ }^{4}$ Etobicoke General Hospital, Etobicoke; ${ }^{5}$ University of Toronto, Toronto, Ontario

Correspondence: Ms Jennifer Boyd, 57 Centre Street, Thornhill, Ontario L4J 1G2. Telephone 905-764-0774, e-mail jenn.boyd@utoronto.ca
} 


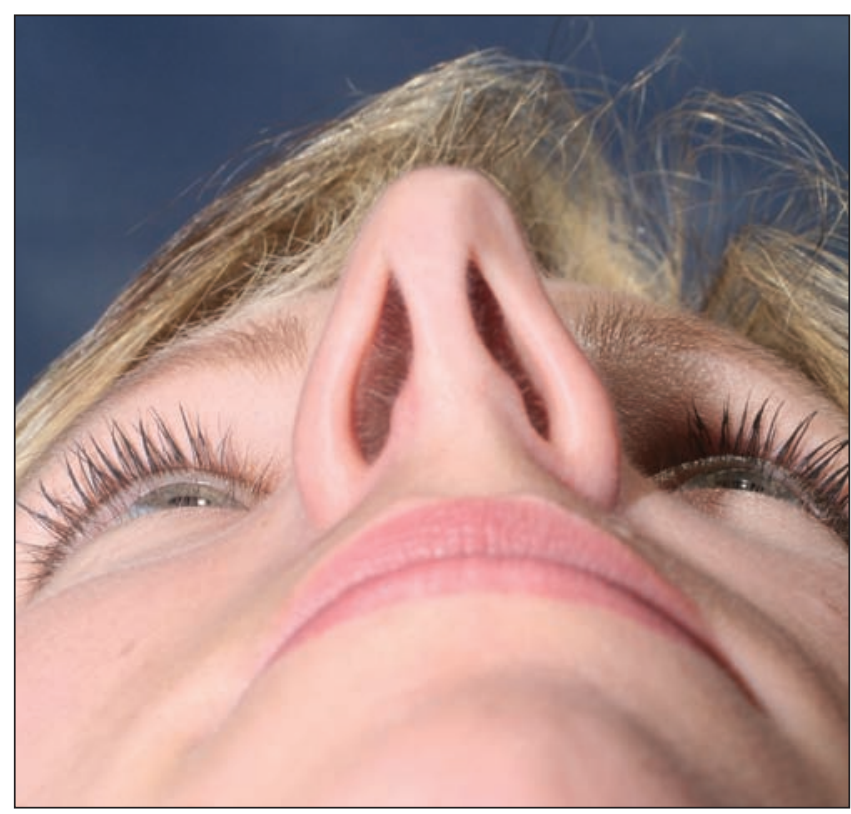

Figure 1) Preoperative 'Pinocchio' deformity

spine, the surrounding skin and soft tissues, and the alar side walls $(2,6)$

There have been several definitions described for nasal tip projection. In the present paper, it is defined as the distance of the nasal tip from the vertical facial plane as measured from the alar crease.

Several methods have been described for the measurement of nasal tip projection. Methods have been described by Simons (7), and Crumley and Lanser (8). Another method commonly used is Goodes' analysis, which states that when the nasofacial angle is between 36 and 40 degrees, the length of the perpendicular line through the tip defining point will be 0.55 to 0.60 of the length of the nasal dorsum (8).

The overprojected nasal tip is a result of an excessive overgrowth of the lower half of the nose (1). The anatomical components that may be overdeveloped include the alar cartilage, septal cartilage and nasal spine (9). However, a deficiency of adjacent structures involving the nasofrontal angle, any saddlenose deformity, mandibular retrusion or anterior displacement of the maxilla may also contribute to an overprojectedappearing nasal tip.

Several techniques of deprojecting the nasal tip have been described and most involve weakening, dividing or excising segments of the alar area. Other less destructive techniques include releasing fibrous attachments, using either the delivery approach, or a full or complete transfixion incision (9). A full transfixion incision disrupts the attachment of the medial crura to the caudal septum, which weakens nasal tip support, resulting in deprojection of the tip. Although this concept has been well described in the literature and studied in conjunction with other tip deprojecting manoeuvres, to date, there have been no studies that have isolated the effect of the full transfixion incision alone.

The purpose of the present study was to determine the effect of the full transfixion incision on tip projection. Specifically, the two questions that were addressed were: what is the quan-
TABLE 1

Reduction of nasal tip projection

\begin{tabular}{llcl}
\hline Group* & $\mathbf{n}$ & Mean, $\mathbf{m m}$ & SD \\
\hline 1 (thin) & 26 & 2.12 & 0.065 \\
2 (medium) & 30 & 1.3 & 0.065 \\
3 (thick) & 17 & 1.41 & 0.507 \\
\hline
\end{tabular}

*Groups are categorized based on skin thickness

titative effect of a full transfixion incision alone on nasal tip projection?; and is there a difference in effect among different nasal skin types?

\section{METHODS}

Seventy-two consecutive patients requesting primary septorhinoplasty procedures were enrolled in the study between January 2003 and February 2004. Patients were recruited from a private otolaryngology practice setting and informed consent was obtained. Patients were included if part of the patient's aesthetic goal was a desire to reduce their nasal tip projection; and the patient was subjectively evaluated by one of the authors (RR or PS) to have an overprojected tip (Figure 1). Patients were excluded if revision procedures were requested. Tip projection was measured from the alar crease to the most prominent point of the nasal tip. Measurements were taken with calipers. The tip was considered projected if tip projection was greater than two-thirds of the length of the nose as a measurement from the nasion to the tip-defining point. Subjects were also categorized into three groups based on the quality of the skin - thin, medium or thick - based on the surgeon's subjective analysis. Nasal tip projection measurements were performed preoperatively and immediately following the full transfixion incision.

All surgical procedures were performed by RR or PS. No local anesthetic was administered before the measurements or the incision. The full transfixion incision was fully taken down the membranous columella to the nasal spine, disrupting the fibrous attachments between the medial crura footplates and caudal septum.

After measurements were taken, the remainder of the rhinoplasty was performed using the graduated approach.

\section{RESULTS}

A total of 73 patients were enrolled in the study, and were comprised of 49 women (67\%) and 24 men (33\%). The mean age was 27 years, ranging from 16 to 55 years of age.

The nasal tip projection was measured in millimetres. Measurements were taken before and immediately after the full transfixion incision was performed and the difference between the two measurements was calculated for each patient. The overall mean nasal tip deprojection of all 73 patients after a full transfixion incision was performed was $1.6 \mathrm{~mm}$, ranging from $0 \mathrm{~mm}$ to $3 \mathrm{~mm}$.

The patients were placed into one of three nasal skin thickness types: thin, medium or thick. There were 26 patients in the thin skin group, 30 patients in the medium skin group, and 17 patients in the thick skin group. The mean \pm SD difference in deprojection was calculated in each group (Table 1). Patients with thin skin had a mean deprojection of $2.12 \pm 0.065 \mathrm{~mm}$. Patients with medium skin thickness had a mean deprojection 
TABLE 2

Post hoc test comparing results of skin thickness quality

\begin{tabular}{|c|c|c|c|c|}
\hline A & B & $\begin{array}{c}\text { Mean (A-B), } \\
\text { mm }\end{array}$ & $\begin{array}{c}\text { Standard } \\
\text { error }\end{array}$ & $\mathbf{P}$ \\
\hline Thin & Medium & 0.815 & 0.1666 & $<0.001^{*}$ \\
\hline Thin & Thick & 0.0704 & 0.1940 & 0.002 \\
\hline Medium & Thick & -0.0112 & 0.01888 & 0.825 \\
\hline
\end{tabular}

${ }^{*}$ Denotes statistical significance

of $1.3 \pm 0.065 \mathrm{~mm}$. For patients with thick skin, the mean nasal tip deprojection was $1.41 \pm 0.507 \mathrm{~mm}$. Overall, patients in the thin skin group had the greatest deprojection from the full transfixion incision compared with the other two groups.

To evaluate if the difference between the groups was statistically significant, a one-way ANOVA was performed. With an F-value of 13.173 , it revealed that there was a significant difference in the mean deprojection among the three groups $(\mathrm{P}<0.0005)$. To further identify which groups were significantly different, a post hoc analysis was performed. (Table 2). This revealed a significant difference between the mean result of the thin skin group compared with both the medium skin and thick skin thickness groups. The difference in deprojection between the medium and thick skin group was not statistically significant. Therefore, not only did the thin skin thickness group have the largest deprojection of the nasal tip with the full transfixion incision, but this was a significant difference compared with the medium and thick skin groups. Figure 2 shows the postoperative result of a septorhinoplasty patient for nasal tip deprojection.

\section{DISCUSSION}

Management of the nasal tip is one of the most challenging goals for a facial plastic surgeon. It has only been in the past 40 years that we have come to understand the important components of nasal tip support. We now know that one of these components is the medial crura and its attachment to the caudal septum (10).

There have been several techniques described to deproject the nasal tip. These techniques include the release of fibrous attachments, decreasing the volume of overdeveloped anatomic components, and repositioning the medial or lateral crura (9). Other methods that give the illusion of a reduced nasal tip by de-emphasizing nasal projection through the augmentation of adjacent structures have also been described (9).

The full or complete transfixion incision is one method of releasing the fibrous attachment to deproject the nasal tip. The medial crura attachment to the caudal septum was identified in 1971 as an important component of nasal tip support (3). Further studies by Petroff et al (10) confirmed that this was important in the postsurgical nose. A complete transfixion incision interrupts both mucosal surfaces and medial crural attachments, allowing the tip to settle closer to the face (9). This technique has been recognized as a useful method to deproject the nasal tip, either alone or with other procedures to correct mild to moderate degrees of overprojection.

Although several studies investigating nasal tip deprojection have included complete transfixion incisions as part of their technique, no study has attempted to qualify the isolated effect of a complete transfixion incision on nasal tip deprojection (10). Although it has been described that patients with

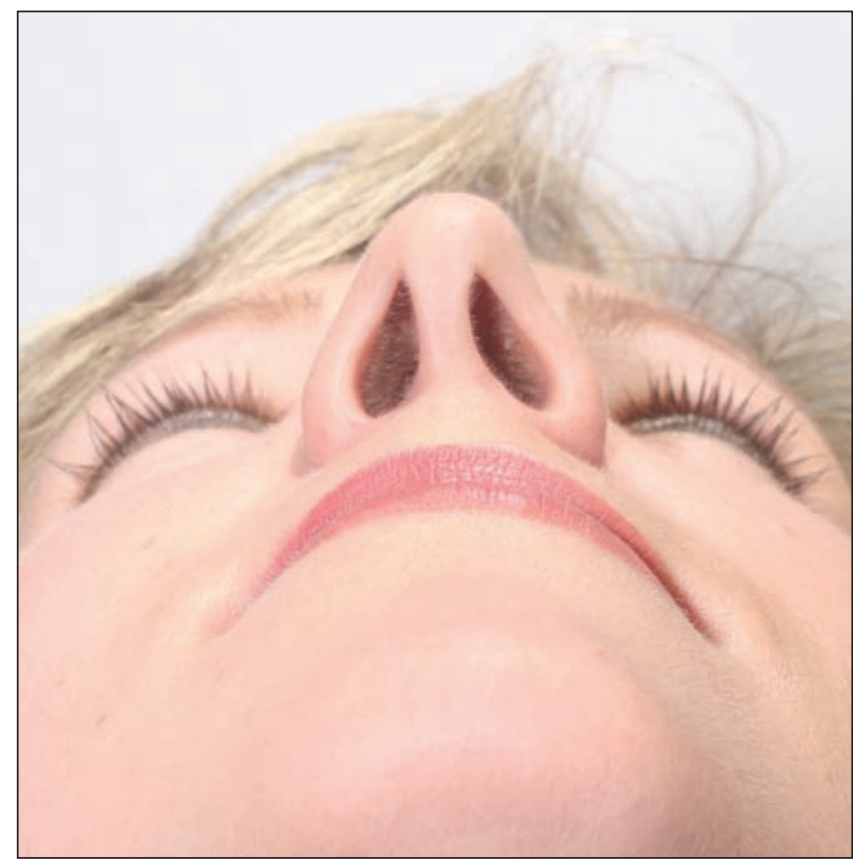

Figure 2) Postoperative result of a septorhinoplasty patient for nasal tip deprojection

differing skin thicknesses may have different results compared with others, there are no studies that have confirmed this.

Unlike other studies that have measured nasal deprojection pre- and postoperatively, in our study, measurements were taken before and immediately after a full transfixion incision was performed. It was believed that this would be the best way to assess the cause and effect of one specific manoeuvre, namely, the full transfixion incision. Furthermore, no local anesthetic was used before the measurements. This was believed to be important because it has been reported (10) that there is an increase in projection of the tip by $1.5 \mathrm{~mm}$ before the procedure begins due to the administration of local anesthetic and manipulation of the nose. All efforts were made to minimally alter or manipulate the nasal anatomy so that true nasal deprojection measurements were performed and were not due to any such artefacts.

We set out to critically assess the impact of the full transfixion incision on nasal tip projection to allow us an objective way to plan nasal tip deprojection surgery. The data in our study support the hypothesis that a full transfixion incision has a quantitative effect on nasal tip deprojection. The overall mean of nasal tip deprojection in our study was $1.6 \mathrm{~mm}$ immediately after the incision was made. This number may be considered small, but is reasonably comparable with other studies, which found an average of $2.4 \mathrm{~mm}$ in tip deprojection when this type of incision was combined with other procedures (10). The thin skin group had a mean deprojection of $2.12 \mathrm{~mm}$, which more closely approximates the average found in combination studies.

Patients were subjectively grouped into different skin thickness types to determine if there was a difference in results based on this characteristic. Patients with thin skin had a larger nasal tip deprojection compared with those with medium or thick skin. Although not a large difference between the groups, the 
results were statistically different. This will aid in surgical planning for patients with mild to moderate overprojected nasal tips who have thin skin, and may reduce the number of manoeuvres required to achieve the aesthetic goal.

\section{CONCLUSIONS}

The study goal, which was to evaluate objectively the effects of the transfixion incision on tip projection, was successful. We were able to quantify the effects of the full transfixion incision

\section{REFERENCES}

1. Fredricks S. Tripod resection for "Pinocchio" nose deformity. Plast Reconstr Surg 1974;53:531-3.

2. Cummings et al, eds. Cummings Otolaryngology - Head and Neck Surgery, 3rd edn. St Louis: Mosby, 1998.

3. Janeke, JB, Wright WK. Studies on the support of the nasal tip. Arch Otolaryng 1971:93:458-64.

4. McCullough E, Mangrat D. Systematic approach to correction of the nasal tip in rhinoplasty. Arch Otolaryngol Head Neck Surg 1981;107:12-6

5. Anderson JR. A reasoned approach to nasal base surgery. Arch Otolaryng 1984;110:349-58. on nasal tip surgery. Also, we were able to demonstrate that patients with thin skin had a greater reduction in tip projection than those with medium or thick skin. The surgeon can thus use his or her subjective assessment of the skin type in planning the surgery and what methods he or she will use. Furthermore, the rhinoplasty surgeon can then use a graduated approach in which the least destructive method (ie, the transfixion incision) is used first and may then use additional manoeuvres if necessary.

6. Beaty MM, Dyer WK, Shawl MW. The quantification of surgical changes in nasal tip support. Arch Facial Plast Surg 2002;4:82-91.

7. Simons R. Nasal tip projection, ptosis and supratip thickening. Ear Nose Throat J 1982;61:454-5.

8. Crumley RL, Lanser M. Quantitative analysis of nasal tip projection. Laryngoscope 1988;98:202-8.

9. Papel ID, Mabrie DC. Deprojecting the nasal profile. Otolaryngol Clin N Am 1999;32:65-87.

10. Petroff MA, McCollough EG, Hom D, Anderson JR. Nasal tip projection: Quantitative changes following rhinoplasty. Arch Otoloaryngol Head Neck Surg 1991;117:783-8. 